

\title{
Louis Jugnet et les réseaux intellectuels catholiques intransigeants. Ce que révèle sa correspondance inédite
} Cyrille Dounot

\section{To cite this version:}

Cyrille Dounot. Louis Jugnet et les réseaux intellectuels catholiques intransigeants. Ce que révèle sa correspondance inédite. Revue d'histoire de l'Eglise de France, 2015, 101, pp.325 - 347. 10.1484/J.RHEF.5.109671 . hal-01398674

\section{HAL Id: hal-01398674 \\ https://hal.uca.fr/hal-01398674}

Submitted on 17 Nov 2016

HAL is a multi-disciplinary open access archive for the deposit and dissemination of scientific research documents, whether they are published or not. The documents may come from teaching and research institutions in France or abroad, or from public or private research centers.
L'archive ouverte pluridisciplinaire HAL, est destinée au dépôt et à la diffusion de documents scientifiques de niveau recherche, publiés ou non, émanant des établissements d'enseignement et de recherche français ou étrangers, des laboratoires publics ou privés. 


\section{LOUIS JUGNET}

\section{ET LES RÉSEAUX INTELLECTUELS}

CATHOLIQUES INTRANSIGEANTS

\section{CE QUE RÉVÈLE SA CORRESPONDANCE INÉDITE}

La correspondance inédite d'un intellectuel est toujours une source d'inestimable valeur pour l'historien. Celle de Louis Jugnet (1913-1973) livre des informations précieuses sur les milieux intellectuels catholiques français. Jugée "considérable » à sa mort ", elle n'a cependant pas subsisté telle quelle ${ }^{2}$ : outre quelques notes manuscrites, les archives privées de $\mathrm{M}^{\mathrm{me}}$ Jugnet ne contiennent malheureusement plus que soixante-huit lettres, essentiellement la correspondance active dont le philosophe gardait trace par copie sur papier carbone (cinquante-sept lettres) ${ }^{3}$. Cette correspondance s'étend du 17 avril 1969 au 27 août $19722^{4}$ et concerne vingt-cinq personnes plus ou moins connues et impliquées dans les milieux intransigeants, tant laïcs que clercs : le $\mathrm{D}^{\mathrm{r}}$ Jean Joublin, Paul Poitevin, Julien Crépin, Jean Oudin, Marcel De Corte, Jean de Bronac, les abbés Pierre Grelot, Noël Barbara, Raymond Dulac, Louis Coache, Georges de Nantes, Duffaud-Riu, Léonidas Chrol (orthodoxe), Angelo Bertuletti, les PP. Ernest Mura, Louis-Marie Simon, o.m.i., RogerThomas Calmel, o.p., Michel-Louis Guérard Des Lauriers, o.p., Victor Sarat, c.p.c.r., dom Jean Roy, dom Gérard Calvet, $\mathrm{M}^{\mathrm{gr}}$ Antonio Romeo, $\mathrm{M}^{\mathrm{gr}}$ Jean Mouisset, évêque de Nice, et les cardinaux Franjo Šeper et John Wright. De loin, le correspondant le plus actif est le père Louis-Marie Simon, qui reçoit quinze lettres de Jugnet. De surcroît, ces lettres sont souvent les plus longues

1. «Bibliographie », dans L'ordre français, $\mathrm{n}^{\circ} 174$ (sept.-oct. 1973), p. 104, n. 1 : « De plus, sa correspondance était considérable. Louis Jugnet correspondait avec de nombreuses personnalités."

2. Louis Jugnet faisait mention d'« une centaine de lettres du père de Tonquédec, qui sont souvent d'un grand intérêt", dans son hommage "Traditionnel et moderne : le R. P. J. de Tonquédec (1869-1962)", dans La pensée catholique, n ${ }^{\circ} 84$ (1963), p. 25. Ces lettres ont malheureusement disparu. Qu'il nous soit permis ici de remercier vivement $\mathrm{M}^{\mathrm{me}}$ Jugnet, qui nous a si gentiment ouvert les portes de sa demeure.

3. Dans une lettre au P. Simon, du 18 mai 1970, il explique à son correspondant l'origine des «traces de carbone, [qui] viennent de [s]on modeste matériel pour garder le double des lettres intéressantes ». Dans les citations qui suivent des lettres de Jugnet, nous imprimons en italique les passages soulignés par lui.

4. Mort le 12 février 1973, et malade bien avant cette date, Jugnet ne prenait plus depuis lors le temps d'écrire, ce qui constituait pour lui un vrai calvaire. 
et les plus précises, mais se rapportent davantage à la crise de l'Église qu'aux réseaux intellectuels. Il est vrai que c'est cette crise qui conduit Jugnet à prendre la plume et à manifester ses liens avec d'autres penseurs ou acteurs du monde catholique, essentiellement dans la mouvance intransigeante ou traditionaliste. Nous avons dit ailleurs quelle part prit Jugnet aux débats liturgiques post-conciliaires ${ }^{5}$, et notre propos actuel est de mettre en lumière la pensée et l'importance de Louis Jugnet d'après ces témoignages inédits.

Louis Jugnet est un philosophe de tradition thomiste, formé à la faculté de Lille ${ }^{6}$. Après l'obtention de l'agrégation, il commence sa carrière professorale à Châteauroux, puis rejoint Toulouse en 1945 ; il y finira ses jours, enseignant à la fois en classes préparatoires au lycée Pierre-de-Fermat et à l'institut d'études politiques. En 1952, il fonde le Cercle Pie-X et donne à partir de cette date de multiples conférences ouvertes aux étudiants, dans lesquelles il affiche ses opinions maurrassiennes et intransigeantes. Pour bien comprendre son insertion dans le monde intellectuel des années post-conciliaires, il faut partir de ses motivations. Celles-ci se trouvent abondamment exposées dans divers documents inédits, qui corroborent ce que nous savons déjà par ses écrits publiés. Les premiers combats intellectuels de Jugnet, menés après-guerre, visent l'évolutionnisme darwinien et ses répercussions philosophiques (à l'encontre de Teilhard de Chardin notamment) ${ }^{7}$, avant qu'il ne s'intéresse plus spécialement à la question de la psychanalyse et fasse connaître en France

5. C. Dounot, “"Pour la résistance catholique.” La participation de Louis Jugnet aux débats liturgiques post-conciliaires, d'après sa correspondance inédite ", dans J.-Fr. GALINIERPallerola, Ph. Foro et A. Laffay (éd.), Les laïcs prennent la parole : la participation des lä̈cs aux débats ecclésiaux après le concile Vatican II (actes du colloque organisé par le Centre Histoire et théologie, laboratoire Histoire, théologie, religions, à l'Institut catholique de Toulouse, du 30 janvier au $1^{\text {er }}$ février 2014), [Paris], 2014, p. 61-93.

6. Il y a soutenu un diplôme d'études supérieures de philosophie, avec un mémoire intitulé Essai sur les rapports entre la philosophie suarézienne de la matière et la pensée de Leibniz. Il écrira à J. Crépin, le 26 juin 1969 : « Je suis un quasi converti de l'adolescence, et [...] c'est par un hasard apparent que j'ai alors découvert la philosophie thomiste, étant un pur produit de l'enseignement universitaire, matériellement parlant."

7. Voir notamment son article fondateur "A propos de l'évolutionnisme catholique », dans $L a$ pensée catholique, $\mathrm{n}^{\circ} 4$ (1947), p. $52-78$ [repris dans Louis Jugnet, cahier $\mathrm{n}^{\circ} 1$ (1975), p. 67-107], critiquant vertement l'article "Transformisme" du Dictionnaire de théologie catholique, dû à $\mathrm{M}^{\mathrm{gr}}$ Amann : «Nous estimons que cet article est fort discutable scientifiquement, inacceptable philosophiquement, et qu'il prête à de grosses réserves du point de vue théologique. " Jugnet publie ensuite «Science allemande, théologie romaine et évolution », dans La pensée catholique, $n^{\circ} 11$ (1949), p. 24-30. En 1951, il adresse une note à $M^{\mathrm{gr}}$ Lusseau, qui est envoyée par ce dernier au P. Mura et transmise au Saint-Office (éditée dans J. DE VIGUERIE, « La crise de l'Église en France dans les années 1950 et 1951, d'après un document inédit ", dans Bulletin de la Société française d'histoire des idées et d'histoire religieuse, $\mathrm{n}^{\circ} 13$ (2003), p. 29-33), dans laquelle il signale comme " point le plus important » les dérives doctrinales « en ce qui concerne les doctrines évolutionnistes ». Il publiera encore "Réflexions sur le teilhardisme », dans L'ordre français, $\mathrm{n}^{\circ} 5$ (mai 1963), p. 37-56 ; "Contre l’idolâtrie teilhardienne", dans Midi étudiant (Fédération générale des étudiants de Toulouse), nouv. série, $\mathrm{n}^{\circ} 1$ (mai 1965), p. 5-6 ; "Teilhard et les incroyants ", dans Itinéraires, t. 108 (déc. 1966), p. 67-75, et «Claudel, saint Thomas et Teilhard ", dans Itinéraires, t. 115 (juil.-août 1967), p. 102-106. Un chapitre de son cours Problèmes et grands courants de la philosophie est consacré à " Teilhard de Chardin » (nouv. éd., [Versailles], 1974, p. 205-214). Une note relative à ce thème, diffusée auprès de ses étudiants, a été publiée après sa mort sous le titre "Deux textes sur Teilhard de Chardin ", dans Louis Jugnet, cahier $\mathrm{n}^{\text {o }} 2$ (1976), p. 79-84. Enfin, Jugnet avait donné une conférence publique, le 10 mars 1960, sur "Teilhardisme et marxisme». 
l'œuvre d'un psychiatre autrichien anti-freudien, Rudolf Allers. Il entretient une correspondance longue et suivie avec ce dernier et rédige un ouvrage présentant sa doctrine ${ }^{8}$. Cependant, avec la crise que subit l'Église, ses engagements prennent une tournure plus extérieure, et plus orientée vers le combat théologique. Jugnet considère cette crise comme la plus grave qu'elle ait jamais connue, "sans équivalent dans l'histoire de l'Église (même l'arianisme ne fait pas le poids en comparaison, pour divers motifs) " ${ }^{9}$. Ce qui l'amène à s'intéresser davantage aux questions purement religieuses.

Il y a un certain paradoxe chez Jugnet quant à l'origine de cette crise de l'Église. D'un côté, il estime que le concile Vatican II est la « source réelle de nos maux " ${ }^{10}$, la " racine » du mal. Il dénonce cette "catastrophe sans nom que fut Vatican II (comme tel, et non par un "détournement" de sa "vraie pensée") " ${ }^{11}$. Mais, d'un autre côté, il voit dans le modernisme la source du mal, contre lequel il mène une lutte sans faille. Il se qualifie volontiers de catholique traditionnel et fait sienne l'expression de Louis Salleron en faveur d'un "catholicisme intact» ${ }^{12}$. C'est au nom de ce catholicisme "le plus classique » qu'il entend résister ${ }^{13}$. Sa correspondance est parsemée d'autorités, puisées chez les Pères de l'Église ou les papes, comme cette allocution de

8. L. Jugnet, Un psychiatre philosophe : Rudolf Allers ou L'anti-Freud, Paris, 1950. La psychanalyse est l'objet de plusieurs articles de Jugnet : «À propos de la psychanalyse », dans $L a$ pensée catholique, no 9 (1949), p. 40-55 ; "Réflexions sur la psychiatrie actuelle », ibid., n 16 (1950), p. 5-21; "Études récentes sur la psychanalyse et le freudisme», ibid., nº 18 (1951), p. 89-92, et "Freudisme et psychanalyse", dans La vie spirituelle : supplément, t. 5 (1951), p. 375-385. Un chapitre de son cours Problèmes et grands courants de la philosophie est consacré à "Freud (1856-1939) et la psychanalyse" (nouv. éd., [Versailles], 1974, p. 118-138). Cette question est abordée dans une lettre au $\mathrm{D}^{\mathrm{r}}$ Joublin, le 14 août 1972 : «Le mot présent est destiné à vous féliciter pour votre si ferme article de La pensée catholique [...] concernant notre commune et vieille ennemie qu'est la psychanalyse. Six n'est qu'un imbécile méchant et prétentieux, mais il fait bien du mal. Salleron l'avait déjà pas mal giflé, mais votre article dégage bien les soubassements freudiens de son inqualifiable navet. Je suis d'accord avec vous sur le fait que la "concupiscentia venerorum" est bien plus consciente qu'on ne le dit ; que la racine fondamentale des préoccupations humaines est métaphysique (cf. R. Allers et V. Frankl, et même H. Baruk, qui a souvent dit d'excellentes choses) ; que le rêve est ambigu, protéiforme, et que l'interprétation psychanalytique est subjective (Pierre Janet, sauf erreur, avait déjà publié une étude intitulée "Une forme nouvelle de paranoïa : la psychanalyse") ; que le succès de cette pseudo-science (succès, hélas, acquis en fait, malgré quelques "outsiders"...) vient d'une mise en condition du public, d'un lavage de cerveau; que la psychanalyse brise les structures mentales de l'homme, détruit la foi et le sens moral. Bref, je tiens à vous dire mon accord sur tout l'essentiel de votre article si opportun. Quant à nous faire entendre, c'est une autre affaire !... » Il est ici question des nombreuses réactions suscitées par le livre de Jean-François Six, La véritable enfance de Thérèse de Lisieux : névrose et sainteté, Paris, 1972.

9. Lettre à J. de Bronac, 23 juillet 1969. À ce sujet, il confie à son interlocuteur : « $\mathbf{M}^{\mathrm{gr}}$ Rupp est vraiment un homme de bien, mais il s'obstine (au moins dans ses propos publics) à minimiser quelque peu la gravité de la crise."

10. Lettre à P. Poitevin, 4 juin 1969.

11. Lettre à J. de Bronac, 23 juillet 1969.

12. L'expression est utilisée lors d'une conférence de février 1953 intitulée «Un auteur d'actualité : Louis Veuillot ».

13. Dès 1947, dans des "Réflexions sur le "nouveau catholicisme" ", éditées dans Louis Jugnet, cahier $n^{\circ} 6$ (1981), p. 13-18, à la p. 17, il s'en prenait aux tentatives des «néo-modernistes [qui] abandonnent la philosophie scolastique ». Il a laissé d'autres articles ou notes sur ce sujet, dont certains ont été publiés après sa mort : "De neo modernismo ou Misère de l'esprit moderniste » (1952), ibid., p. 49-53 ; "Sur la caractérologie du moderniste et du libéral » (1952), ibid., p. 57-58 ; "Esquisse sur la psychologie moderniste : "animalia quae modernistae dicuntur..." " (1953), ibid., p. 59-62, et « Face au modernisme », dans Itinéraires, n ${ }^{\circ} 86$ (sept.-oct. 1964), p. 39-54. 
Pie X du 27 mai 1914, reproduite plusieurs fois : «Prétendre concilier la foi avec l'esprit moderne conduit non seulement à la diminution, mais à la perte totale de la foi."

Le problème essentiel du modernisme tient, selon Jugnet, à ce qu'il n'a pas été assez combattu, permettant à cette hérésie de reparaître sous une forme nouvelle et insolente ${ }^{14}$. Il dénonce "le néo-modernisme omniprésent " et rappelle que son combat, ancien, est bien antérieur à Vatican II :

«De la situation de l'Église, rien à vous apprendre : pendant plus de vingt ans, et même un quart de siècle bientôt, nous avons été quelques-uns à annoncer l'essentiel de ce qui risquait d'arriver. On ne nous a, somme toute, pas écouté (même sous Pie XII). Voyez les résultats ${ }^{15}$ !»

Écrivant la genèse de cette crise majeure, Jugnet déplore l’ épouvantable responsabilité » de la hiérarchie, qui, "même sous Pie XII, [...] n’a somme toute, à la différence de saint $\mathrm{Pie} \mathrm{X}$, pris que des "mesurettes" répressives " 16. Il confie à ses interlocuteurs qu'il "prie pour ceux qui combattent le néomodernisme » ${ }^{17}$, complimente le père Calmel pour son «dernier article [...] contre le néo-modernisme " ${ }^{18}$, et, de manière habituelle, approuve tous ceux qui mènent une "énergique résistance à l'invasion moderniste dans le haut clergé " ${ }^{19}$. Dans une lettre au père Grelot, il fait aussi voir que sa réaction, celle d'un laïc, est aussi dirigée contre le monopole clérical, celui des «nouveaux prêtres " ${ }^{20}$. Reprochant à son ami d'enfance de ne s'attaquer qu'aux traditionalistes, et de le faire dans Le Figaro (« Tu écris dans les journaux bourgeois !»), il s'insurge contre une pensée unique, seule libre d'antenne :

«Tu sais bien que des théologiens réputés (et souvent surfaits, à mon sens) nient équivalemment les supposés fondamentaux du salut — je ne te ferai pas un dessin...

14. Lettre au cardinal Seper, 15 juillet 1969 : « Chaque jour, revues et journaux nous apportent des déclarations insolentes, et souvent hérétiques, de clercs modernistes et progressistes auxquels on ne demande aucune espèce de rétractation : un Hans Küng, un Schillebeeckx peuvent impunément se permettre à longueur d'année de menacer Rome de schisme, et professer les idées les plus aberrantes sur la Trinité, l'Incarnation, le Péché originel, la Rédemption ou l'Eucharistie, sans jamais être frappés d'aucune sanction. "

15. Lettre au P. Mura, 30 décembre 1969.

16. Lettre à J. Oudin, 3 juin 1969 : «Que dire de la situation ? J'en reste à l'excellente formule de $\mathrm{M}^{\mathrm{gr}}$ Rodhain (autour de janvier) : “La crise ne fait que commencer." Dieu sait pourtant qu'elle est étendue et profonde. Maintenant, tout le monde le proclame de haut en bas de l'Église. J'ai tout vu venir depuis les années 1946, et, lorsqu'on me traitait de paranoïaque ou de visionnaire, je répondais ironiquement par ce passage du Dies irae : Quidquid latet apparebit... Je crois que maintenant les plus gourdes sont au parfum... Et la hiérarchie porte en l'occurrence une épouvantable responsabilité, même sous Pie XII, qui n'a somme toute, à la différence de saint Pie X, pris que des "mesurettes" répressives. De Jean XXIII, mieux vaut ne pas parler (il faudrait être Dante). Quant au pape régnant, s'il pousse de fréquents cris d'alarmes (dont les modernistes se foutent "usque ad mortem"), il les assortit fréquemment de louanges au concile et au sain aggiornamento. Or c'est la racine qui est mauvaise. "

17. Lettre à l'abbé Duffaud-Riu, 12 avril 1970.

18. Lettre au P. Calmel, 8 juillet 1969 : «Vous touchez là l'essentiel. " Il s'agit de l'article "Passion et Résurrection : mystères de grâce ", dans Itinéraires, no 135 (juil.-août 1969), p. 175-193, spécialement « le paragraphe en petits caractères (p. 187-193) ».

19. Lettre à M. De Corte, 9 juin 1969.

20. La référence implicite au roman de Michel de Saint-Pierre, paru en 1964, vient souligner son désarroi face aux nouveautés liturgiques ou doctrinales. 
—, et qu'une très notable partie du jeune clergé, et même du moins jeune, dit des énormités doctrinales. Mais tu réserves les coups de ton fouet à ceux qui gueulent un peu en sens inverse. Est-ce de leur faute si on n'a admis à parler à l'ORTF, et ce, pour des raisons sur lesquelles j'ai mon avis, qu' un porte-parole qui "primarise" tout, alors qu'il y a, je tiens à te le rappeler, à l'étranger comme en France, des gens compétents (théologiens ou philosophes) auxquels on ferme résolument la porte au nez en toute occasion? Car la théologie dite nouvelle (même quand elle ressasse des vieilleries multiséculaires) a pour elle les grandes maisons d'édition catholique, la grande presse catholique, l'ORTF, etc. Ceci, tout le monde ne le voit que trop bien ${ }^{21}$ !»

Jugnet dresse des constats effarants quant à la révolution qui s'opère au sein de l'Eglise, notamment lors de la Semaine sainte de 1970 : «Tout s'y est passé en dépit des normes : les élèves du séminaire romain ont menacé de ne pas assister à la messe du jeudi saint parce qu'elle était trop longue (sic). Le Vendredi saint, l'après-midi, ils sont allés assister à un match de rugby. Le pape, disant sa messe, a sauté systématiquement toutes (il n'en reste déjà guère) les formules concernant les saints, etc. ${ }^{22}$." Toutefois, de manière générale, sa correspondance ne cible que rarement les modernistes, prélats ou théologiens. Il vise Guitton, qui va, dans Le Monde, «s'attendrir sur le souvenir du grand Loisy » ${ }^{23}$, ou encore fait référence aux propos d'un "prélat romain des mieux orientés » au début du concile : "Les meneurs de jeu, ce ne sont pas Suenens et Liénard, ni Alfrink, Döpfner, etc. Ce sont Béa et Montini." Je cite, sans commenter ${ }^{24}$."

Ses principaux correspondants, qui sont parfois ses adversaires, sont des gens issus du milieu traditionaliste. Ces personnages constituent aussi la plupart de ses sujets de préoccupation. Aussi, après avoir montré ce que pense Louis Jugnet, nous tenterons d'éclairer sa place dans le traditionalisme français de l'après-concile. Nous serons largement servis par le genre épistolaire, puisque, comme il l'indique: «J'ai pris la ferme résolution de poursuivre inébranlablement ma tâche doctrinale auprès des gens que Dieu m'a confiés, y compris les lecteurs éventuels d'articles et les correspondants français ou étrangers ${ }^{25}$. "Malgré ses réticences, Jugnet prend alors fréquemment la plume : «Moi qui n'écris guère, et qui n'aime pas les lettres à tel ou tel, je me lance ces temps-ci dans ce genre de campagne, tant je suis excédé des âneries dites, écrites ou faites par certains ${ }^{26}$."

21. Lettre au P. Grelot, 4 mai 1971.

22. Lettre au P. Simon, 18 mai 1970.

23. Lettre à J. de Bronac, 23 juillet 1969.

24. Ibid.

25. Lettre à J. Oudin, 3 juin 1969.

26. Lettre à l'abbé Dulac, 29 juin 1969. À la mi-juillet, il confesse : « Pour ma part, je fais une véritable campagne de lettres, très étudiées, pour encourager les uns, documenter les autres, couler tels autres, etc. J'y passe mes journées depuis trois semaines " (lettre au P. Barbara, 16 juillet 1969). 


\section{La pensée de Louis Jugnet}

La figure de Jugnet est celle d'un traditionaliste qui ne tergiverse pas sur les termes. Il fait profession d'un «catholicisme traditionnel», ou intransigeant ${ }^{27}$. Il se qualifie à plusieurs reprises de «traditionnel " ${ }^{28}$. Il parle des « catholiques traditionnels » ${ }^{29}$, des « revues traditionnelles » ${ }^{30}$, des "théologiens traditionnels " 31 , des "prêtres traditionnels » ${ }^{32}$ ou des "religieux traditionnels " ${ }^{33}$, voire des «tradis " ${ }^{34}$. Il évoque la "vraie théologie traditionnelle » ${ }^{35}$, et même «l'Église traditionnelle » ${ }^{36}$. Mais le plus fréquemment il emploie seulement l'adjectif substantivé de "traditionnels" ${ }^{37}$.

Contrairement à Jean Madiran, qui réfute vivement le terme d'intégriste, et en écrit l'histoire ${ }^{38}$, Jugnet ne s'en offusque pas outre mesure ${ }^{39}$. Il le prend souvent à son compte dans sa correspondance privée pour désigner la mouvance traditionnelle, en y distinguant les intégristes « mous » des intégristes "durs », et en se rangeant dans la dernière catégorie ${ }^{40}$. Aussi écrit-il au père Calmel : "Nous autres, je veux dire les intégristes "durs" [... ${ }^{41}$.» De la même manière, il fustige « les soi-disant intégristes de modèle courant " ${ }^{42}$, ces "pauvres cocus intégristes " ${ }^{43}$, encore appelés "les "cosidett $i^{\prime "}[$ sic] intégristes » ${ }^{44}$. Il allègue les «publications et mouvements dits "intégristes" " 45 , évoque le cas d'un de ses anciens étudiants, ex-séminariste «parti pour crime d'intégrisme " ${ }^{46}$, fait référence au " climat "intégriste" courant " ${ }^{47}$ ou, tout simplement, aux « intégristes » ${ }^{48}$. Jugnet cependant entoure quasi systémati-

27. Il parle aussi de ceux "qu'on eût nommé au siècle dernier les "zelanti" " (lettre à P. Poitevin, 4 juin 1969).

28. Dans une lettre au P. Chrol du 9 juin 1971, il recommande d'aller voir le bâtonnier Georges Boyer, "catholique traditionnel comme [lui]".

29. Lettre à l'abbé de Nantes, 9 juin 1969.

30. Lettre au P. Simon, 2 juin 1969.

31. Lettre à M. De Corte, 12 juillet 1969.

32. Lettre à l'abbé Dulac, 29 juin 1969.

33. Lettre au P. Simon, 26 avril 1970.

34. Lettre au P. Simon, 6 mars 1972.

35. Lettre à J. Oudin, 5 juillet 1969.

36. Lettre à P. Poitevin, 4 juin 1969.

37. Ces variations se retrouvent quarante-quatre fois dans sa correspondance.

38. J. Madiran, L'intégrisme : histoire d'une histoire, Paris, 1964 (Coll. Itinéraires, 15).

39. On dénombre vingt et un emplois de ce terme dans sa correspondance.

40. Il signale à l'abbé de Nantes, dans une lettre du 30 juin 1969, que « la cassure s'accentue entre intégristes "durs" et "mous" ". Le 5 juillet 1969, il écrit à J. Oudin : "Il y a actuellement un clivage entre intégristes "durs" et "mous". Pour ma part, j’ai choisi depuis longtemps, car je voyais venir ! " Oudin lui répondra, le 17 juillet suivant : «Finalement, mon intégrisme se durcit, comme le vôtre, cher vieux camarade. " Au P. Barbara, dans une lettre du 16 juillet 1969, il fait mention "de forts noyaux "durs" très décidés ", rencontrés par le P. Simon, o.m.i., en Provence.

41. Lettre au P. Calmel, 8 juillet 1969.

42. Lettre à l'abbé de Nantes, 16 juillet 1969.

43. Lettre à Jean de Bronac, 23 juillet 1969.

44. Lettre à J. Oudin, 3 juin 1969.

45. Lettre au P. Simon, 6 mars 1972.

46. Lettre à l'abbé Dulac, 29 juin 1969.

47. Lettre à dom Roy, père abbé de Fontgombault, juillet 1969.

48. Dans un article antérieur, "Le père Congar et l'intégrisme ", dans La pensée catholique, $\mathrm{n}^{\circ} 15$ (1950), p. 127-128, Jugnet exposait ses raisons d'être "intégriste ": "Penser que saint Thomas a dit tout l'essentiel sur un sujet est [pour le P. Congar] signe d'intégrisme ! La chose est 
quement ce terme de guillemets, prenant ses distances avec l'acception courante, tout en conservant son utilité pratique ${ }^{49}$.

Il est à noter que Jugnet, qui ne parle presque jamais de politique ${ }^{50}$, trace ici un parallèle par le biais du terme d'intégriste. En 1969, il écrit à Poitevin : «Je ne puis absolument pas admettre qu'on porte maintenant ses efforts contre une extrême droite intégriste, réputée dangereuse, hétérodoxe même (car, enfin, c'est bien ce que vous dites), alors qu'avec des maladresses fort humaines elle dit des choses très justes pour l'essentiel. " En 1970, dans une lettre au P. Simon, il rend compte des Journées maurrassiennes organisées par la faculté de droit d'Aix-en-Provence, où il remarque un «haut niveau intellectuel et... [un] "intégrisme" généralisé»" ${ }^{11}$. Pour Jugnet, ce côté politique est sous-jacent, en partie lié au spirituel, comme il le reconnaît dans cette même lettre : «Ceci est, en principe, plus politique que religieux, mais il y a, vous le savez, une symbiose, ou une synergie, des deux facteurs ", tout comme le dira plus tard le père Calmel ${ }^{52}$. Il écrit d'ailleurs : «Comme on a

belle, car Léon XIII se voit ainsi ex consequenti ranger sous ce prestigieux vocable ; n'a-t-il pas dit textuellement : “Il n'est point de problème posé devant la conscience moderne qui ne trouve dans saint Thomas souvent la solution juste et adéquate, toujours les principes nécessaires pour le résoudre"? De même, on reste confondu lorsqu'on voit défini l'intégrisme - cette erreur si pernicieuse - par le désir "de façonner ou déterminer tous les domaines de la vie à partir de l'élément spécifiquement et proprement catholique". Quel mal y a-t-il à vouloir que la Vérité éclaire tous les recoins du réel ?"

49. En 1966, dans "Quelques réflexions sur Le paysan de la Garonne de J. Maritain », édité dans Louis Jugnet, cahier n ${ }^{\circ} 6$ (1981), p. 90, il utilisait l'expression « ce qu'on nomme [...] "un intégriste" ", "sans du reste jamais pouvoir en fournir une définition rigoureuse et incontestable ». Dès 1953, dans une conférence sur Veuillot, il remarquait : « Il faudrait d'abord donner une définition de l'intégrisme, et ce mot ne se trouve dans aucun document romain... » Il concluait sa conférence par une plaisanterie sur «l'intégrisme, du nom d'Intègre, hérésiarque fameux qui n'a sans doute pas existé, mais dont la doctrine fait néanmoins des ravages ".

50. On trouve seulement quelques allusions à ses agissements à Sciences-Po Toulouse. Ainsi, dans une lettre au P. Simon du 26 avril 1970 : «J'ai l'honneur d'être attaqué dans une feuille gauchiste pour m'être rué (c'est le mot qu'ils emploient) sur un "commando" subversif qui cherchait à envahir un local universitaire, et pour en avoir sérieusement "cogné" deux membres. " Le 3 décembre 1970, il écrit à l'abbé Duffaud-Riu : «Nous avons eu une matinée assez pénible à l'IEP : menace réalisée de raid gauchiste violent, pas de police (ou sans droit d'intervention intérieure), et un personnel comportant quatre hommes, de combativité variable. Je ne puis ni ne souhaite présentement vous raconter l'affaire, pour des raisons diverses. Je pense du reste que les troubles et les menaces vont continuer, et ceci à divers signes. Dieu merci, un afflux étonnant de jeunes accourus pour ma protection, plus un amphi favorable en sa totalité de fait (à part le ou les mouchards gauchistes ou du PC de service). Bien simplement, je tiens à force de volonté et par la grâce de Dieu, mais je commence à m'user : je mange peu, je dors très peu et mal. “On" veut nous avoir par ce biais. Il y avait déjà le travail très lourd, la lutte doctrinale ; il y a maintenant, de nouveau, des problèmes de sécurité pure et simple. De telle manière que, présentement, je ne puis pratiquement voir personne. " On ne trouve rien d'autre, sinon de simples allusions éparses aux "gauchistes ".

51. Lettre au P. Simon, 26 avril 1970.

52. R.-Th. CALmel, Les mystères du royaume de la grâce, t. I, Les dogmes, $3^{\mathrm{e}}$ éd., Poitiers, 2013 [éd. orig. Jarzé, 1972], p. 13-14 : «Si le théologien dans toute l’immense zone de l'expérience et de la connaissance de l'humain par connaturalité affective est accordé, sans même bien le savoir, avec les mythes révolutionnaires et rousseauistes, avec une certaine vision irréelle de l'homme, une conception messianique et évolutionniste de la cité, bref, si le théologien dans tout une part de son intelligence, et la part quelquefois la plus secrète et la plus personnelle, est en accord avec les erreurs modernes, avec les aberrations condamnées par le Syllabus et Pascendi, alors sa théologie, serait-elle correcte sur beaucoup de points, pâtira peu ou prou, mais nécessairement, de cette déviation cachée. [...] Les thèses modernistes, qui se répandent depuis le concile 
démoli mon cours de philosophie politique (trop engagé...), on l'a remplacé par un cours de philosophie pure à l'IEP (Les grands courants philosophiques). Vous pensez de quelle façon je mène l'affaire! Ainsi, je puis reprendre à la racine même les principales erreurs actuelles ${ }^{53}$. "

Du point de vue politique, Jugnet est un fidèle maurrassien, qui n'a aucune empathie pour l'Allemagne et ses erreurs : il fait grief à Guitton, «cet ex-collabo ", d'avoir écrit pour La Gerbe ${ }^{54}$; il se rit des antisémites de son camp, de leur attitude paradoxalement "judaïque » 55 ou de leurs "arguties rabbiniques " 56 ; il déplore l'insistance des clercs sur l’obéissance ("Vous savez pourtant où celle-ci a mené l'Allemagne » ${ }^{57}$ ); il juge sévèrement les revues faisant «l'éloge de traîtres (au sens univoque), comme Rebatet (voir Les décombres !) » ${ }^{58}$. Faisant partie de ces catholiques blessés par la condamnation de l'Action française (1926) puis soulagés par sa levée (1939), il craint que les sanctions portées contre l'abbé de Nantes ne soient une nouvelle répression injuste, "une réédition du coup de 1926 " ${ }^{59}$. Jugnet a défendu maintes fois le penseur de l'Action française, du point de vue politique et philosophique, notamment dans ses articles « Charles Maurras et la philosophie " 60 et "Le réalisme de Charles Maurras » ${ }^{61}$.

Sa correspondance avec Paul Poitevin est révélatrice de ce point de vue ${ }^{62}$. Contre les imputations de naturalisme, il réplique : « Je trouve vos conclusions trop rapides et beaucoup trop sévères pour Maurras (ce qui, vous le savez, m'est toujours très sensible). [...] D'abord, il ne faut pas surestimer la dose de positivisme chez Maurras [...]. Maurras, au fond, était presque aristotélicien,

Vatican II, sont commandées partiellement par une volonté révolutionnaire de transmutation de la cité $[. .$.$] . C'est [...] un immense malheur pour la cité, et ce n'est pas une bénédiction pour le$ théologien, lorsque le même savant personnage qui est, ou veut être, thomiste en spéculation pure accepte d'être libéral ou socialiste en politique et freudien en psychologie. Que théologie et politique demeurent distinctes, rien de plus normal ; encore faut-il que chez le même auteur ces deux disciplines ne se fassent pas une guerre latente."

53. Lettre à J. Oudin, 3 juin 1969.

54. Lettre à Jean de Bronac, 23 juillet 1969.

55. Lettre à J. Oudin, 3 juin 1969 : «Une des maladies intégristes est le millénarisme, erreur doctrinale bien nommée, et d'origine spécifiquement judaïque : pour des gens le plus souvent antisémites, ça ne manque pas de pittoresque !"

56. Lettre au P. Simon, 23 juin 1970.

57. Lettre au P. Sarat, 8 juillet 1969. Il contestait l'usage fait par ce prêtre, «pour illustrer [sa] conception, d'un officier allemand pendant la dernière guerre mondiale, à cause de son "obéissance" ".

58. Lettre au P. Simon, 6 mars 1972. Jugnet y fait référence à la revue Lecture et tradition.

59. Lettre à J. de Bronac, 23 juillet 1969.

60. Article paru dans La pensée catholique, $\mathrm{n}^{\circ} 115-116$ (1968), p. 62-71. Cet article a été critiqué par G. WaIlliez, "Charles Maurras et la philosophie », dans Cahiers Charles Maurras, $\mathrm{n}^{\circ} 34$ (avr.-juin 1970), p. 44-47. Jugnet y a répliqué, sous le même titre, dans Cahiers Charles Maurras, $\mathrm{n}^{\circ} 36$ (oct.-déc. 1970), p. 28-30 (son article est suivi, p. 29-30, des remarques de G. Wailliez).

61. Article paru dans L'ordre français, $\mathrm{n}^{\circ} 174$ (sept.-oct. 1973), p. 100-103 [repris dans Actes du deuxième colloque Maurras "Tendances et perspectives des études maurassiennes " (Aixen-Provence, Institut d'études politiques, 31 mars, $1^{\text {er }}$ et 2 avril 1970), Aix-en-Provence, 1973 (Études maurrassiennes, 2), p. 117-119].

62. Paul Poitevin fut secrétaire général de la fédération internationale Una voce jusqu’à sa démission le 25 janvier 1970, en signe de protestation contre l'acceptation du Bref examen critique. Cette fédération, créée en 1964, s'est donnée pour mission de promouvoir le chant grégorien et la polyphonie sacrée, puis le missel de saint Pie V. 
de plus en plus en vieillissant ${ }^{63}$. "Sa conclusion est sans appel : «En outre, je suis toujours peiné par les poussées anti-maurrassiennes qui se produisent périodiquement même chez les meilleurs des "intégristes" : nous avons vraiment mieux à faire que d'aller critiquer Maurras et son "naturalisme" en 1969 !»

Dans sa correspondance, c'est à travers la politique qu'il aborde la philosophie, relativement à Maritain. Jugnet n'est pas un détracteur de Maritain, bien au contraire. Il s'oppose à lui sur plus d'un point, c'est indéniable, mais, loin de le dénigrer systématiquement, comme font d'autres écrivains traditionalistes, il s’en fait le débiteur. Il écrit par exemple à Crépin, en 1969 :

«Parlons maintenant un peu de Maritain : en un sens, je lui dois beaucoup, puisque ses grands ouvrages de ce temps-là furent ma source et mon guide, conjointement avec ceux des regrettés pères de Tonquédec et Garrigou-Lagrange (dont le Dieu, notamment, reste un chef-d'œuvre insurpassé). J'ai rencontré Maritain à diverses reprises, et il fut toujours très accueillant pour moi. Je le tiens pour un homme de très grande valeur. Reste qu'outre un certain nombre de points sur lesquels je n'accepte pas ses vues (notamment les rapports entre art et morale, entre éthique naturelle et théologie, ou encore la conception existentielle du thomisme, ou la fameuse "motion brisable" du Court traité), je suis profondément opposé à toute sa philosophie politique (ainsi, $\mathrm{du}$ reste, qu'un certain nombre d'autres thomistes actuels, canadiens, espagnols, italiens et autres). De telle manière — si on y ajoute sa solitude volontaire dans sa retraite — que je ne l'ai pas revu depuis 1939. Je pense souvent à lui, je crois qu'il ne m'a pas oublié, mais nous en restons là ${ }^{64}$."

63. Lettre à P. Poitevin, 17 avril 1969. Il poursuit un véritable réquisitoire : « Saint Thomas dit à peu près mot pour mot les mêmes choses qui vous ont rebuté dans l'article de Pujo ! Si l'homme est bon ontologiquement (comme tout ce qui est, comme tel, puisque "ens et bonum convertuntur"), s'il est une image de Dieu, s'il a gardé des éléments positifs dans l'intelligence et la volonté (ceci contre Luther, Calvin et les jansénistes), le mal moral en est statistiquement le plus facile et le plus fréquent dans son comportement. Lisez $\mathrm{I}^{\mathrm{a}}$, q. 63 , a. 9, ad $\mathrm{I}^{\mathrm{um}}$, et $\mathrm{I}^{\mathrm{a}} \mathrm{II}^{\mathrm{ae}}$, q. 71, a. 2 , ad $3^{\text {um }}$." A J. Oudin, le 5 juillet 1969, il rapporte cet échange de vues et dénonce " une attaque oblique contre la pensée maurrassienne et son "naturalisme" vers le printemps (quel sens des urgences et de l'opportunité !) ».

64. Lettre à J. Crépin, 26 juin 1969. Cette lettre reprend ce qu'il écrivait deux ans plus tôt, dans "Jacques Maritain et le néo-modernisme ", dans La pensée catholique, $\mathrm{n}^{\circ} 107$ (1967), p. 53-61, à la p. 53 : «C'est presque à contrecœur que nous rédigeons cet article, car il nous place dans une situation assez pénible : nous risquons de mécontenter un certain nombre de nos amis, dont les uns reprocheront d'être trop favorable à l'ouvrage de Maritain [Le paysan de la Garonne], et les autres, d'être trop réservé envers lui. C'est qu'en effet nous ne pouvons ni ne voulons oublier tout ce que nous devons à Jacques Maritain, dont l'œuvre nous fut connue à partir de 1930, dès le début de notre vie d'étudiant, en même temps que celle du père Garrigou-Lagrange et du père de Tonquédec. Par la suite, nous avons rencontré quelques fois Jacques Maritain, qui nous montra beaucoup d'affabilité et de bonté. Mais c'est surtout la lecture très attentive de ses livres qui nous a enrichi. Disons plutôt de ses ouvrages de philosophie pure, car nous sommes en total désaccord avec son œuvre politico-sociale. Comment ne pas rappeler ici ce que furent, et ce que sont restés, pour tant d'entre nous, Trois réformateurs, Antimoderne, Réflexions sur l'intelligence et La philosophie bergsonienne? Mais nous sommes en totale opposition avec ses vues sur la démocratie, l'histoire, etc. " Il avait jugé, en 1966, que Maritain " est tout le contraire d'un homme de droite », précisant : "Depuis les années 1926, lors de l'affaire de l'Action française, il n'a cessé d'œuvrer dans le sens de l'ouverture à gauche, et son action a eu, en particulier, des effets aussi étendus que profonds dans le clergé et l'intelligentsia d'Amérique latine. Sa philosophie politique est un essai de synthèse entre l'idéologie de la Révolution française et la pensée de saint Thomas d'Aquin. Disons en passant combien nous sommes opposés pour notre part à cette partie de son œuvre, ainsi du reste que de nombreux thomistes français ou étrangers. Mais ceci est une autre histoire..." ("Quelques réflexions sur Le paysan de la Garonne... », p. 90). 
Cette distinction essentielle entre les parties métaphysique et politique de l'œuvre de Maritain se retrouve quand il s'agit de juger de Paul VI, convaincu d'être "maritanien sur tout le plan politico-social (voyez son scandaleux discours de naguère à l'ONU, comparé à l'Église par certains côtés) " 65 .

La formation intellectuelle de Jugnet est un élément essentiel de compréhension. Du point de vue philosophique, il s'affiche purement thomiste : «Je suis thomiste de l'espèce "dure", et je dois l'essentiel de mon orientation aux regrettés pères J. de Tonquédec et Garrigou-Lagrange, si injustement méconnus actuellement ${ }^{66}$. " Il écrira peu après : «Je suis effectivement thomiste strict, par une attention spéciale de la Providence [...]. Je suis un quasi converti de l'adolescence, et [...] c'est par un hasard apparent que j'ai alors découvert la philosophie thomiste, étant un pur produit de l'enseignement universitaire, matériellement parlant ${ }^{67}$. » En philosophe réaliste, il juge « que l'idéalisme est le péché philosophique par excellence, qu'il est la somme de toutes les erreurs ou presque, qu'on ne doit pas lui faire la moindre concession [...], et qu'il y a chez saint Thomas tous les éléments nécessaires à sa discussion, soit dans le Commentaire sur la Métaphysique (voir Cathala $\mathrm{n}^{\mathrm{o}} 637$, 661-662, 705-707, 712-713 et 1800), soit dans la I', q. 85, a. 2, resp., Secundo, quia..., dont la richesse est virtuellement illimitée ».

Jugnet cite souvent ses maîtres, les déplorés pères de Tonquédec ${ }^{68}$ et Garrigou-Lagrange ${ }^{69}$. En plus de Maritain, il évoque d'autres philosophes avec des réserves plus ou moins grandes, comme Étienne Gilson, le père Sertillanges, Régis Jolivet ${ }^{70}$ ou Simone Weil ${ }^{71}$.

65. Lettre à P. Poitevin, 17 avril 1969.

66. Lettre à J. Crépin, 30 mai 1969. Il écrira de même au cardinal Wright, dans une lettre du 16 juillet 1969: "Je suis thomiste, disciple des regrettés pères de Tonquédec et GarrigouLagrange, et j'ai publié, notamment, une introduction à saint Thomas qui me valut alors une lettre très bienveillante de celui qui était en ce temps-là le cardinal Montini. »

67. Lettre à J. Crépin, 26 juin 1969.

68. «Que j’ai connu de fort près, et dont j’ai gardé un gros paquet de lettres personnelles ; je ne fais aucune réserve doctrinale ni méthodologique» (ibid.). Il écrit à J. Oudin, le 19 juillet 1969 : «Par ailleurs, je n’aime pas Marcel Clément, qui fut jadis odieux envers le très regretté père de Tonquédec. " En 1972, dans sa dernière conférence, "Le réalisme catholique ", il donnait cet ultime témoignage d'estime : « Le père de Tonquédec, qui était un homme très traditionnel mais extrêmement mesuré et qui ne voyait pas de l'infaillibilité du pape partout, et surtout où il n'y en a pas, m’a dit un jour : “Pascendi, je crois que ça engage l’infaillibilité du magistère." Moi, qui étais déjà anti-moderniste, j’étais tout content, mais je lui demandai : "Et pourquoi ?" Il me répondit: "D'abord à cause de l'aspect fondamental de son objet, de son contenu, et puis, ensuite, à cause de l'extrême solennité des formules employées ; on y sent un homme qui n'agit pas en tant que personne privée, mais en tant que pontife suprême, et qui mise à fond sur ce qu'il dit." Il est en effet parfaitement possible que l'infaillibilité soit engagée dans une encyclique et, qu'inversement, elle ne le soit pas dans un concile" (cité dans "Bibliographie commentée des œuvres de Louis Jugnet ", dans Le sel de la terre, n 47 (hiver 2003-2004), p. 214).

69. Lettre à J. Crépin, 26 juin 1969.

70. Il déplore, quant à l'idéalisme, « de malencontreuses formules du P. Sertillanges, et même, vers la fin, de Jolivet » (ibid.).

71. Lettre à $\mathbf{M}^{\text {gr }}$ Romeo, 30 juin 1969 : «En ce qui concerne Simone Weil, je suis, pour ma part, assez réservé : je ne connais qu'une partie de ses écrits, mais, quelle que soit la générosité de caractère de cette fille, et la droiture de ses intentions, sa conversion au catholicisme est des plus ambiguës, voire douteuse. Tâchez de retrouver un article de Marcel Moré [« La pensée religieuse de Simone Weil ", dans Dieu vivant, n 17 (oct.-déc. 1950), p. 35-68] [...]. La thèse de l'auteur est que $\mathrm{S}$. Weil était, au fond, manichéenne, cathare. La même interprétation est, du reste, défendue 
Il n'aborde qu'assez peu les problèmes philosophiques dans sa correspondance, seulement lorsque cela sert de fondement à une critique théologique. Ainsi écrit-il à son ami d'enfance le père Grelot : « Je ne suis pas d'accord avec ta problématique et tes présupposés philosophiques (à mon sens, tu donnes trop aux sciences de la nature, et plus encore à des philosophies qui — et là, je suis chez moi, puisque j'étudie cette discipline en spécialité depuis 1930 sont des plus aléatoires à mon avis) ${ }^{72}$. " Dans une autre lettre, il ajoute ces quelques lignes :

«Il est assez clair que pour toi "la haine de l'hellénisme est le commencement de la sagesse", alors que je suis tout à l'inverse (si tu veux souffrir, mais comprendre mieux ce que je veux dire, lis avec simplicité l'étude de mon vieux co-équipier Marcel De Corte, "Aristotélisme et christianisme", in Archivio di filosofia, 1954, I [repris dans La philosophie de l'histoire de la philosophie, Rome/Paris, 1956 (Bibl. d'histoire de la philosophie), p. 81-97] [...]. Les théologiens de pointe refont, en fait, ce qu'on fait les "archaïsants" du xvir siècle : retour (et que ça saute!) à la sainte Écriture et aux Pères (saint Augustin!), mais conceptualisons le tout avec la philosophie nouvelle (Descartes, grand Dieu ! Maintenant, ce sera Heidegger ou un autre) ${ }^{73}$."

Jugnet n'est cependant pas en consonance totale avec les autres thomistes. Marcel Clément en fait les frais. Son jugement sur les débuts de la Faculté libre de philosophie exprime son inquiétude quant à l'orientation philosophique de ce nouvel établissement ${ }^{74}$. Et ses revirements politiques le rendent encore plus suspect aux yeux de Jugnet ${ }^{75}$.

par le néo-cathare Déodat Roché (Cahiers cathares de 1951). Pour ma part, je trouve que certains catholiques traditionnels se sont trop enthousiasmés pour cet auteur, de pensée très ambivalente."

72. Lettre au P. Grelot, 4 mai 1971. Son attrait pour l'exégèse l'avait porté à lire « attentivement " les ouvrages du P. Grelot. Il rappelle à son correspondant : « Je sais surtout qu'en dehors de l'exactitude scientifique d'une traduction il y a son impact sur le troupeau du Christ. Le regretté père Lagrange, lui, ne l'oubliait pas lorsqu'il écrivait : "La critique n'est pas tout, il y a la discipline des âmes." Si donc la traduction litigieuse peut invoquer certains arguments en sa faveur, c'est par rapport à une psychologie et à un vocabulaire qui ne sont pas ceux de l'homme occidental ordinaire, pour lequel pourtant (du moins on le dit) on a refait la liturgie. " Ailleurs, il traite du P. Feuillet, p.s.s., en des termes faisant voir sa connaissance du sujet : "Je tiens celui-ci pour un vrai savant et un homme de foi, à tout ce qu'il semble. Cependant, n'est-ce pas l'Introduction à l'Écriture sainte de Robert et Feuillet qui avait été si fermement "épinglée" par le vaillant Antonino R[omeo] et par la Congrégation des études? En outre, cette interprétation du thème de la Parousie aboutit quand même à convaincre d'erreur les apôtres et les premières générations chrétiennes. Est-ce bien conforme à la doctrine traditionnelle (voir les anciennes controverses dans Drach-Bissing, l'intervention de la Commission biblique "in illo tempore", etc...) ?» (lettre au P. Simon, 23 décembre 1970). Il remerciera par la suite le P. Simon de sa " si nette et si théologique mise au point concernant l"article "Parousie" de M. Robert », ajoutant : " Il est certain qu'il n'y a plus d'exégèse théologique maintenant, à part celle des "survivants" du Latran, et de vétérans comme $\mathrm{M}^{\mathrm{gr}}$ Spadafora [...]. La notion de "fides quaerens intellectum" est pratiquement évacuée par les modernes, même modérés " (lettre au P. Simon, ${ }^{\text {er }}$ mars 1971).

73. Lettre au P. Grelot, 28 juin 1971.

74. Lettre à J. Oudin, 19 juillet 1969 : « Deux mots, maintenant, sur la nouvelle Faculté libre de philosophie en gestation (dont personne ne m'a jamais envoyé le moindre avis, ni demandé quoi que ce soit : tout ce que j'en sais, c'est par ouï-dire ; ce n'est pas mon amour-propre qui en souffre — Dieu seul peut évaluer à quel point les questions de ce genre m'indiffèrent —, mais j’y vois déjà un signe de l'orientation future...)."

75. Lettre à l'abbé Duffaud-Riu, 12 avril 1970 : «Marcel Clément, apologiste de Maurras lors de la mort de celui-ci (j'ai des textes sous les yeux), puis ennemi de la pensée maurrassienne (il y 
Il en ira de même avec le cardinal Journet, pourtant assez bien apprécié des milieux traditionalistes :

«Vous n'avez pas l'air d'adorer cet espoir suprême de certains traditionnels. Comme vous le situez bien! Déjà, le regretté "Luc-Verus" disait de son Église du Verbe incarné que son véritable inspirateur était "non pas saint Thomas, mais J. Maritain". Mais il y a plus : je vais vous montrer, pièces en main, la malhonnêteté insigne du bon cardinal ${ }^{76}$.»

Abordons maintenant ces réseaux dans lesquels Louis Jugnet s'investit, parfois au risque de les malmener.

\section{Les réseaux de Louis Jugnet}

Jugnet est assez sévère sur son milieu et ne tape que subsidiairement à gauche contre «les réformateurs iconoclastes " ${ }^{77}$. Il s' occupe principalement de ce qu'il appelle assez trivialement les « conneries innombrables » du camp " intégriste ": "chasse aux révélations privées folkloriques (je me suis fait engueuler pour mon opposition décidée à Garabandal, San Damiano, NotreDame-de-Tous-les-Peuples, etc.), [...] actes de servilité, ou même de simple modérantisme excessif devant la sacro-sainte "hiérarchie", etc. " ${ }^{78}$. Il pense que les problèmes majeurs tiennent au manque de formation générale $\mathrm{du}$ laïcat et au caractère difficile des personnes dotées d'une bonne formation : "On constate toujours les mêmes carences intellectuelles ou caractérielles chez les gens de nos milieux ${ }^{79}$. » Il précise : «Du reste, il y a trop de laïques du second ordre dénués des "habitus" théologiques, et qui dogmatisent en ce domaine [la messe] dans nos rangs : ça augmente la confusion ${ }^{80}$."

a deux naturalismes politiques, l'un statique, celui de Maurras, l'autre mobiliste, celui de Marx : deux erreurs symétriques)."

76. Ibid. : « Dans L’Église du Verbe incarné, t. I, éd. de 1941, l’auteur est amené à envisager le problème de la guerre sainte et à se demander si celle-ci est bien fondée théologiquement, si ceux qui y participent peuvent être dits martyrs, etc... On peut lire (p. 382, n. 2) que "saint Thomas rappellera au contraire (de l'islam) que l'Église ne vénère pas comme martyrs ceux qui sont morts les armes à la main" ( $\left.\mathrm{II}^{\mathrm{a}} \mathrm{II}^{\mathrm{ae}}, 124,5,3\right)$. Bien! Mais alors, lisez le texte (l'art. 3 en sa totalité : "Tamen, quia")... Rajoutez y le Suppl., 96, 6, ad 11 (c'est tout de même du saint Thomas !). Mais continuons : p. 391 et 394, alors que la question reparaît, il fait le "black-out" total sur les textes si clairs de saint Thomas concernant les ordres militaires (cf. $\mathrm{II}^{\mathrm{a}} \mathrm{II}^{\mathrm{ae}}$, q. 188 , a. 3), si utiles pour nous, je crois, aujourd'hui plus que jamais. Plus précisément, p. 391, n. 1, il parle effectivement des ordres militaires, mais sans parler de la doctrine thomiste à leur sujet!, et, p. 394, il ne renvoie sur cette question qu'à $\mathrm{II}^{\mathrm{a}} \mathrm{II}^{\mathrm{ae}}$, q. 40, a. 2, "Utrum clericis [in genere!] et episcopis liceat pugnare". N'est-ce pas là l'équivalent d'un faux, une tromperie (qui, à mon sens, ne saurait être involontaire) du lecteur?"

77. Lettre à P. Poitevin, 4 juin 1969.

78. Lettre à J. Oudin, 3 juin 1969.

79. Lettre au P. Simon, 2 juin 1969.

80. Lettre au P. Simon, 6 mars 1972. En sens inverse, il se réjouit de certaines interventions épistolaires, comme celles de Bernard Faÿ, de Jean Oudin et des siennes, "qu'on ne peut pas faire passer pour l'œuvre de chaisières illettrées ! " (lettre à J. Oudin, 19 juillet 1969). 
L'on retrouve cet état d'esprit dans une lettre à l'abbé Dulac : «Il est pourtant clair que nous avons la théologie pour nous (encore faut-il en savoir vraiment quelque chose, ce qui n'est pas souvent le cas de ces messieurs !) ${ }^{81}$.» Quand Jugnet parle de la sorte, il ne s'érige pas en modèle, quoi qu'il soit bien informé. Il lit ordinairement les travaux les plus récents en philosophie, théologie, exégèse, fussent-ils en langue étrangère. Ainsi félicite-t-il le R. P. Angelo Bertuletti, le 13 juillet 1970, de son "remarquable travail sur la présence réelle ", une thèse de théologie soutenue au Latran ${ }^{82}$. De même, il fait l'éloge de la thèse de l'abbé Bruno Salleron, Matière et corps du Christ chez saint Athanase d'Alexandrie, "un travail très sérieux et vraiment instructif » 83 .

L'embarras de Jugnet tient aux nombreuses dissensions au sein de la mouvance traditionaliste, et aux luttes intestines stériles. Ses rapports avec cette mouvance, comme ses jugements, vont évoluer avec le temps dans le sens d'un mécontentement grandissant, au point qu'en mars 1972 il dira : "Des publications et mouvements dits "intégristes", je ne suis pas content dans l'ensemble, excepté par Forts dans la foi et le bulletin de l'abbé Coache ${ }^{84}$. " Essayons de détailler ces rapports conflictuels.

En 1969, Jugnet soutient totalement les prises de position publiques de l'abbé Raymond Dulac, rédacteur de Courrier de Rome ${ }^{85}$. Il lui écrit à la fin du mois de juin : «Tout d'abord, recevez l'expression de ma totale adhésion à ce que vous écrivez dans le dernier Courrier : il est temps — grand temps ! de "refuser" 86 ..." Néanmoins, il ne peut s'empêcher d'annoter une lettre de ce dernier critiquant l'abbé Coache : «C'est injuste ${ }^{87}$. " Fin octobre, il estime "le dernier Courrier [...] excellent", tout en commençant à prendre ses distances avec l'auteur : «Je n'ai pas voulu le lui écrire, car il ne prend pas bien les remarques critiques ${ }^{88}$. " Inéluctablement, Jugnet se détache de l'abbé Dulac, à cause de ses médisances envers l'abbé Coache. En mai 1970, il va jusqu'à parler de "l'actuelle inutilité du Courrier de Rome, qui comporte moitié de lamentations stériles, moitié d'invraisemblables arguties rabbiniques pour nous tirer (?) de la situation actuelle », affirmant : «L'abbé Dulac,

81. Lettre à l'abbé Dulac, 29 juin 1969.

82. Lettre au R. P. don Angelo Bertuletti, 13 juillet 1970. L'ouvrage en question est la thèse de l'auteur, La presenza di Cristo nel sacramento dell'Eucaristia, Rome, 1969 (Corona Lateranensis, 19). Il ajoute : «Votre ouvrage me paraît de toute première valeur : d'abord par une orthodoxie rigoureuse, ce qui devient une sorte de miracle dans l'état actuel de la pensée ecclésiastique. Ensuite, par le magnifique "habitus" qui guide vos investigations de théologie spéculative, en particulier sur le concept de présence, et sur le "respectus habitudo" du Christ aux espèces consacrées. Je pense que presque personne ne réalise de tels travaux actuellement..."

83. Lettre au P. Simon, 23 décembre 1970. La thèse de l'abbé Salleron avait été publiée à Rome et Saint-Cénéré en 1967.

84. Lettre au P. Simon, 6 mars 1972.

85. Lettre à l'abbé Dulac, 3 juin 1969 : «Je lis toujours avec autant de contentement, et en esprit d'adhésion totale, le Courrier de Rome. Combien avez-vous raison de dénoncer, de plus en plus clairement, la racine de tous nos maux actuels ! Le lendemain, il décrit à P. Poitevin « Dulac lui-même, si solide et si rigoureux en ses argumentations ».

86. Lettre à l'abbé Dulac, 29 juin 1969.

87. Lettre de l'abbé Dulac, 17 juillet 1969.

88. Lettre au P. Simon, 27 octobre 1969. 
c'est bien net, a atteint le seuil de sa résistance à la "hiérarchie", et n'ira pas plus loin : il en rabattra plutôt ${ }^{89}$."

En 1969, Jugnet collabore encore à La pensée catholique, comme il le fait depuis 1947 90. Début 1970, accablé de travail, il avoue «renoncer à écrire cette année le moindre article de revue, malgré mes promesses à l'abbé Lefèvre ", directeur de la revue ${ }^{91}$. En septembre de la même année, il se rend compte que sa "cote a baissé à La pensée catholique» ${ }^{92}$. Il fait principalement deux griefs à son directeur, l'un doctrinal, l'autre diplomatique :

«L'abbé Luc Lefèvre s'obstine à croire en Paul VI ; et il connaît maintenant surtout par des tiers, car je n'ai pas voulu le heurter de front : il est assez âgé, et pas des plus faciles (encore un!) - mes positions exactes. Après m'avoir promis de faire un petit article sur mon récent cours imprimé (qu'il a depuis février environ) iln'en a rien fait, et n'a même pas répondu à une lettre d'il y a plus de deux mois où je lui disais mes regrets. Tout ce que la revue a fait, c'est de mettre le titre sur une couverture, et de commander un paquet d'exemplaires. En fait, il a des dadas, parisiens de préférence [...]. "La province", mon Dieu, ça vient après. Je n'ai donc pas l'intention d'écrire prochainement un article pour La pensée catholique - gardez ceci pour vous, bien entendu."

En mars 1971, Jugnet constate qu'il est " "vidè" de La pensée catholique par l'abbé Luc J. Lefèvre, qui a enterré le compte rendu de [s]on petit livre, ne répond plus à [s]es lettres depuis plus d'un an (treize mois exactement) et ment à [s]es amis parisiens en disant qu'il [lui] écrit. »Il ajoute : «En fait, ça se voit "comme le nez au milieu de la figure", ça lui a pris dès mes déclarations "pro-Coache" (publiques). [...] De mon côté, j'ai décidé, même si des choses "tournaient", de ne plus reprendre ma collaboration à La pensée catholique, qui continue de prêcher la confiance en Paul VI. [...] Humainement parlant, je suis maintenant réduit au silence, à part mon enseignement universitaire (lequel est, au fond, l'essentiel) ${ }^{93}$. "Un an plus tard, Jugnet affirme qu'il est " totalement éliminé » de La pensée catholique, devenue "l'organe d'un très petit clan »: "Mais c'est bien la faute de l'abbé Luc Lefèvre, qui se veut toujours "papiste" à tout crin ${ }^{94} \ldots$... Il fera de nouveau ce constat : "Je suis "vidé", pour mon compte, depuis pas mal de temps (because le nouvel Ordo missae : je suis de "tranche dure"...) ${ }^{95}$."

Dès le mois de juin 1969, il exprime ses réserves vis-à-vis de certaines figures de l'intransigeantisme, desquelles il s'écarte progressivement ${ }^{96}$. Il se tient en retrait de Jean Ousset, estimant que « La Cité catholique ne se mouille

89. Lettre au P. Simon, 23 juin 1970.

90. Fondée par les abbés Lefèvre, Berto, Lusseau et Roul, cette revue rassemble les «partisans maurrassiens du P. Le Floch», directeur du Séminaire français de Rome. Voir P. Airiau, "Le Séminaire français de Rome ", dans Histoire et Missions chrétiennes, nº 10 (juin 2009), p. 48.

91. Lettre au P. Simon, 30 janvier 1970.

92. Lettre au P. Simon, 7 septembre 1970.

93. Lettre au P. Simon, $1^{\text {er }}$ mars 1971.

94. Lettre au P. Simon, 6 mars 1972.

95. Lettre au $\mathrm{D}^{\mathrm{r}}$ Joublin, 14 août 1972.

96. Sur ce milieu, voir P. Arriau, "Les hommes de La pensée catholique», dans Catholica, nº 60 (été 1998), p. 59-74. 
guère " ${ }^{97}$. Il juge que les mobiles des intransigeants «sont un mélange de trouille et de soumissionisme clérical (il en reste toujours quelque chose) " ${ }^{98}$. Il déplore évidemment « qu'Ousset refuse formellement de prendre position sur le nouvel Ordo missae " ${ }^{99}$. Mais il juge positivement ce repli sur le "socio-politique ": "En un sens, la situation me paraît plus claire à cet égard. » Il gardera ainsi avec La Cité catholique des rapports " diplomatiques [...] assez bons", bien que ce mouvement se tienne "à l'écart du conflit religieux, et [qu'il ait] poliment refusé de participer au colloque de Lausanne, malgré certaines insistances » 100 .

Il en ira différemment des pères de Chabeuil, avec qui il rompt totalement dès l'introduction du nouveau missel. En réponse aux prises de position de la revue Marchons des Coopérateurs paroissiaux du Christ-Roi (CPCR) ${ }^{101}$ dans leur numéro de juin 1969, Jugnet écrit au supérieur, le père Rathelot, pour lui demander " de suspendre l'envoi » de la revue ${ }^{102}$. Il dénonce « la ligne nouvelle adoptée par [son] ordre et [sa] publication », les "formules ambiguës, voire inacceptables », et fait part de son «franc et total désaccord avec [son] attitude d'ensemble : [leur] étroite conception de l'obéissance est en opposition formelle avec le comportement connu de certains Pères et Docteurs de l'Église, "verbo, scripto et opere" ". Le même jour, il signale à Marcel De Corte cet article «particulièrement odieux [...], qui révèle les ravages d'un certain cul-bénitisme » : «Moi qui écris fort peu aux gens et aux journaux, je vais leur envoyer dès aujourd'hui une lettre d'engueulade, avec ordre formel de ne plus m'envoyer leur torchon pieusard. Ne ménageons rien, ni personne ${ }^{103}$. »

Le cas d'Una voce est quelque peu différent, plus attaché à la personne du secrétaire général, « dont le modérantisme et la totale inefficacité crèvent les yeux depuis longtemps ${ }^{104}$... » L'intransigeant le plus malmené par Jugnet est cet "ami déjà ancien, l'ingénieur parisien [Paul] Poitevin, assez versé en liturgie ", avec qui il rompt " complètement » en juin 1969, en raison de son attitude : ce dernier «écrit sans cesse à tout le monde pour morigéner cha-

97. Lettre au P. Simon, 2 juin 1969. La Cité catholique est un mouvement fondé par Jean Ousset en 1946 dans le but de former des militants laïcs en vue d'instaurer un ordre social chrétien, épaulé par la revue Verbe. Ce mouvement réunit « les courants catholiques maurrassiens et les courants catholiques intransigeants ", selon A. FERRON, "Intégrisme et traditionalisme catholique : réflexions autour de la réactivation d'un mouvement contre-révolutionnaire ", dans J. BAudouin et Ph. Portier (dir.), Le mouvement catholique français à l'épreuve de la pluralité : enquête autour d'une militance éclatée, Rennes, 2002 (Des sociétés), p. 182. Voir l'étude approfondie de R. DE Neuville, Jean Ousset et La Cité catholique, Bouère, 1998.

98. Lettre à M. De Corte, 9 juin 1969.

99. Lettre au P. Simon, 18 mai 1970.

100. Lettre au P. Simon, 6 mars 1972.

101. Cette congrégation religieuse, fondée en 1928 par le père Vallet, s'est spécialisée dans la prédication des Exercices spirituels de saint Ignace. Paul Airiau, dans L'Église et l'Apocalypse $d u X I X^{e}$ siècle à nos jours, Paris, 2000 (Faits et représentations), p. 47 et suiv., y voit un exemple marquant des liens créés entre l'apocalyptisme et l'ignatisme. Elle sera liée à La pensée catholique et à La Cité catholique de Jean Ousset.

102. Lettre au père supérieur des CPCR, 9 juin 1969.

103. Lettre à M. De Corte, 9 juin 1969.

104. Ibid. Il y qualifie Poitevin d'《éminence grise»: "C'est lui qui tire réellement les ficelles. " 
cun » ${ }^{105}$. Ailleurs, il le juge «mal équilibré ("caractériel" évident) et d'une insupportable suffisance en matière doctrinale, alors qu'il n'y connaît, en fait, pas grand-chose " 106 .

Autre tête de turc de Jugnet, celle de Pierre Debray, dont il rapporte certains propos hostiles aux traditionalistes ${ }^{107}$. En 1971, Jugnet met en garde ses amis contre cet ancien compagnon de route du Parti communiste. Il s'indigne contre cet «ex-progressiste, intelligence médiocre, infatué de luimême, jaloux, intrigant et manipulé comme une marionnette par le sinistre Daniélou, de "l'Alliance mondiale des religions" (présidée par Maryse Choisy), qui joue les repentis et les traditionalistes " ${ }^{108}$. Et il incrimine le "“mouvement des Silencieux" (ô combien !) " 109. La publication du disque de l'assemblée des Silencieux est pour lui l'occasion de railler le directeur de La pensée catholique : «M. Lefèvre faisait le duettiste du lamentable Pierre Debray. " Jugnet ira jusqu'à qualifier le mouvement des Silencieux, en 1972, de "fléau national $\mathrm{n}^{\circ} 1$ des catholiques traditionnels ». Et d'ajouter : "Il constitue un grand péril pour notre résistance (la "voie de garage"...) ${ }^{110}$."

Jugnet s'écarte lentement mais sûrement d'un autre des tenants de la tradition, Jean Madiran ${ }^{111}$. Début juin, Jugnet s'emporte contre le directeur d'Itinéraires, qui «a eu de vilains procédés contre les deux héroïques prêtres itinérants [Coache et Nantes] qui mènent la lutte contre le catéchisme hérétique " ${ }^{112}$. Une semaine plus tard, il rejette son attitude " proprement ahurissante, car, malgré ses invectives aux évêques, il "fausse et retient", en mettant des bâtons dans les roues à cette héroïque équipe de prêtres " ${ }^{113}$. À la fin du

105. Ibid.

106. Lettre au P. Simon, 27 octobre 1969.

107. Lettre au P. Simon, 6 mars 1972 : « Nous avons évolué... Nous devons nous ouvrir... Nous ne pouvons faire un tri dans ce que dit le magistère... Nous ne possédons pas la vérité. »

108. Lettre au P. Simon, $1^{\text {er }}$ mars 1971.

109. Sur ce mouvement, voir J.-Fr. Galinier-Pallerola, « Le Rassemblement des Silencieux de l'Église, Pierre Debray et le combat liturgique », dans ID., P. Foro et A. LAffay (éd.), Les lä̈cs prennent la parole..., p. 203-240.

110. Lettre au P. Simon, 6 mars 1972.

111. Jugnet avait collaboré occasionnellement à Itinéraires de 1964 à 1967 : « La pression de communs amis m'avait amené à écrire quelques articles (quatre ou cinq en tout) dans Itinéraires, mais ça n'a pas pu marcher longtemps, avec ce genre de citoyen (c'est moi qui ai déclaré les rapports rompus, mais à la suite de mauvais procédés de sa part) " (lettre à l'abbé Duffaud-Riu, 12 avril 1970). Sur Jean Madiran, voir D. Masson, Jean Madiran, Maule, 1989. La revue Itinéraires (1956-1996), principal foyer de convergence intellectuelle des traditionalistes, a été de ce fait « condamnée " par l'épiscopat français. Le 23 juin 1966 paraissait une « Mise en garde des cardinaux et du conseil permanent de l'épiscopat français " (publiée dans La documentation catholique, 17 juillet 1966, col. 1285-1288), considérant « comme un devoir de mettre en garde les fidèles contre des articles parus notamment dans des magazines comme Le monde et la vie, des revues comme Itinéraires et Défense du foyer, des bulletins comme Lumière ».

112. Lettre au P. Simon, 2 juin 1969. L'abbé Coache, curé de Montjavoult (Oise), est rédacteur du bulletin Le combat de la foi et l'organisateur de grandes processions de la Fête-Dieu, interdites par l'ordinaire du lieu en 1968. Sur ce prêtre, voir Gr. CÉLIER, « L'abbé Louis Coache : le combattant de la foi ", article publié en ligne, à l'adresse : http://www.gregoirecelier.fr/Media/ AbbeLouisCoache-1920-1994.pdf [site consulté le 19 août 2015]. L'abbé de Nantes, supérieur d'une communauté fondée à Saint-Parres-lès-Vaudes (Aube), est frappé de suspense ab officio (1963) puis a divinis (1966). Ils coorganisent à partir de l'été 1968 une tournée visant à promouvoir leur action de "résistance ".

113. Lettre à M. De Corte, 9 juin 1969. 
mois du juin, il dénonce la « gaffe » qu'il commet « actuellement en "livrant" (il n'y a pas d'autre mot) les prêtres traditionnels les plus en flèche à notre indigne épiscopat " ${ }^{114}$. En juillet, il impute les attaques de Madiran contre l'abbé de Nantes à « une pure et simple mesquine jalousie personnelle » et imagine les réactions du directeur d'Itinéraires : "On va me prendre mon public! Je ne suis plus l'oracle sur son trépied ${ }^{115}$ !». Il parle ailleurs de "véritable canaillerie " ${ }^{116}$, d' "agression (c'est le seul mot qui convienne) de Madiran contre l'équipe Barbara-Coache-de Nantes " ${ }^{117}$, de « coup de pied de l'âne " 118 , de " coup de poignard dans le dos " 119. Ce que Jugnet reproche essentiellement à Madiran, c'est de prétendre devenir le centre de cette "résistance catholique » : "En fait, Ousset et lui deviennent fous de prétention, ils veulent tout diriger, tout contrôler ${ }^{120}$. "

Les jugements personnels que Jugnet porte sur Madiran sont plus sévères encore en 1970. Il trouve que "Itinéraires rend des services, mais [que] Madiran est proprement "imbuvable" ". Il liste ses défauts, orgueil et " suffisance insupportable ", soulignée déjà par Maurras ${ }^{121}$; il ironise sur ses pseudonymes ${ }^{122}$. Cependant, il ne l'attaque jamais ad hominem en public, ne voulant pas qu'une dépréciation de sa personne entraînât celle de sa pensée. C'est pourquoi il concède au mois de juin que, si ses dispositions à son égard "ne sont pas des meilleures », il n’en pense pas moins que "Madiran est un des rares à tenir fermement » ${ }^{123}$.

114. Lettre à l'abbé Dulac, 29 juin 1969. Est-ce en réaction à cette "gaffe » que Madiran demandera à ses lecteurs, dans le numéro de novembre 1969 d'Itinéraires de ne surtout pas dresser de listes de prêtres disant la messe traditionnelle?

115. Lettre à J. Oudin, 5 juillet 1969.

116. Lettre à J. de Bronac, 8 juillet 1969.

117. Lettre au P. Calmel, 8 juillet 1969. Il poursuit : « Je la sentais venir, et j’étais au courant de pas mal de mauvais procédés déjà du tandem Ousset-Madiran contre ces prêtres héroïques, véritables confesseurs de la foi. Beaucoup de traits, vous le savez, me rebutent chez Madiran [...], mais ne pourriez-vous essayer de lui faire saisir le caractère scandaleux des pages 6-10 du numéro de juillet [d'Itinéraires], où les intéressés sont CALOMNIEUSEMENT accusés de vouloir constituer une sorte d'ersatz du magistère, ou de hiérarchie parallèle, fondant ainsi quelque chose qui “de droit et de fait" serait "une secte" [...]. Beaucoup en ont assez d'être invités sans cesse à se soumettre à ce chef providentiel qu'est... Jean Ousset [...]. Je ne vois pas pourquoi ce primaire pontifiant, et souvent mesquin en ses procédés, devrait être notre nouvelle Jeanne d'Arc ! Il est vrai que Madiran est incapable d'écouter la moindre critique venant de qui que ce soit... »

118. Lettre au P. Barbara, 16 juillet 1969.

119. Lettre à J. Oudin, 19 juillet 1969.

120. Lettre au P. Barbara, 16 juillet 1969. Jugnet se moquera, dans une lettre à l'abbé Duffaud-Riu le 12 avril 1970, des variations du tandem Ousset-Madiran, qui prit du plomb dans l'aile du fait que "Ousset refuse absolument de prendre parti dans l'affaire de la messe " : "Madiran boudant (et jalousant) Ousset, Madiran idolâtre d'Ousset, Madiran brouillé avec Ousset (à suivre)."

121. Lettre à l'abbé Duffaud-Riu, 12 avril 1970 : «D’une suffisance, d'abord, insupportable ("Moi, je", malgré tant de pieuses formules). Maurras avait fort bien vu lorsque, passant en revue peu avant sa mort les jeunes qui pourraient prendre la relève, il cita, entre autres, M. Arfel (vrai nom de Madiran) : "Mais il est trop orgueilleux." "

122. Ibid. : "Il fut Arfel, "inconditionnel" de Maurras et du très regretté père J. de Tonquédec, puis Lagor (même orientation), au point d'en être parfois agaçant. Puis, devenu Madiran (c'est une localité ; à quand Bécon-les-Bruyères ou Les Aubrais ?). " Cette pique est assez surprenante chez celui qui a pris lui-même pour pseudonyme Charnay, village où vécut sa famille (voir M. Brignol, "Confession d'un disciple », dans Le sel de la terre, n 47 (hiver 2003-2004), p. 174).

123. Lettre au P. Simon, 23 juin 1970. 
En définitive, Jugnet refuse à tout prix l'embrigadement des forces traditionnelles sous la houlette unique d'un homme ou d'une institution. Au père abbé de Fontgombault, il résume sa position : «L'action de résistance doctrinale n'a absolument pas besoin de se caporaliser ainsi : elle gagne même en efficacité à rester en partie une action de francs-tireurs, partant de la part des hommes de doctrine qui ne veulent pas être embrigadés ${ }^{124}$. " Dans le même sens, il diffuse les propos du « franc-tireur » Marcel De Corte : «Je crois que la multiplication des points de résistance (surtout dans le clergé) (c'est moi qui souligne) est essentielle ${ }^{125}$.»

Cet « homme qui enseigne depuis tant d'années la morale, [s]on ami Marcel De Corte " ${ }^{126}$, est très lié à Jugnet, lequel ne cesse de vanter ses mérites, et pour qui ses avis ont "du poids " ${ }^{127}$. Leur estime réciproque se profile à travers leur échange de lettres, et elle sera rendue publique à la mort de Jugnet ${ }^{128}$. Ce dernier s'inquiète auprès de son correspondant belge des tensions apparaissant dans le milieu intransigeant (" une partie notable des "traditionnels" s'effraie de nos audaces») et des menées visant tel ou tel prêtre : "Ils sont là toute une bande qui fait tout pour faire échouer l'actuelle campagne de conférences contre le nouveau catéchisme et la liturgie "nouvelle" 129 . " Auprès de ses correspondants, il loue De Corte d'être le seul laïc à dire ouvertement la vérité ${ }^{130}$, et de mener le bon combat par la plume ${ }^{131}$. Jugnet s'autorise de ses jugements et partage ses réactions ${ }^{132}$. Ultime preuve de cette amitié sans faille : la dernière lettre de Jugnet, adressée à dom Gérard, par laquelle il fait ses recommandations à son ami liégeois ${ }^{133}$.

D'autres auteurs sont évoqués en passant. Jugnet entre en contact avec Jean Borella, " agrégé de philosophie, professeur aux classes d'écoles, et totalement dans nos vues ». Au père Simon il écrit : «Je vous l'indique comme contact

124. Lettre à dom Roy, juillet 1969.

125. Lettre de M. De Corte, 16 juillet 1969, citée dans une lettre à J. Oudin, 19 juillet 1969.

126. Lettre au P. Sarat, 8 juillet 1969. Marcel De Corte (1905-1994), ancien élève étranger de l'École normale supérieure, fut professeur de philosophie à l'université de Liège, dont il fut également le recteur. Jugnet l'appelle «mon vieux co-équipier » (lettre au P. Grelot, 28 juin 1971). Ces deux philosophes partagent l'essentiel du point de vue tant de la philosophie thomiste que des idées politiques ou des goûts littéraires (notamment Jean de La Varende ; voir P. Dolley (éd.), L'œuvre de La Varende devant l'opinion : 125 témoignages, Rouen, 1952). Il lui fait part de leur concordance des vues : « Je viens de lire, à l'instant, votre excellent article [...]. Vous êtes en plein dans la bonne voie " (lettre à M. De Corte, 9 juin 1969), et insiste sur son estime : «Vous savez le prix que j'attache à votre approbation et à votre amitié " (lettre à M. De Corte, 12 juillet 1969).

127. Lettre à J. Oudin, 19 juillet 1969.

128. Outre l'article laudateur "In memoriam Louis Jugnet " dans L'ordre français, $\mathrm{n}^{\circ} 174$ (sept.-oct. 1973), p. 24-29, De Corte publiera une préface élogieuse de l'édition posthume des Problèmes et grands courants de la philosophie, [Versailles], 1974.

129. Lettre à M. De Corte, 9 juin 1969.

130. Lettre à J. de Bronac, 8 juillet 1969, au sujet des prêtres réfractaires : «Mais est-ce leur faute si aucun laïc, sauf Marcel De Corte, avec lequel je suis très lié, n'a osé dire ce qu'ils disent? ?, et lettre à J. Oudin, 3 juin 1969 : «Les seuls qui disent toute la vérité sur ce point, ce sont De Corte, G. de N. et Dulac. Les autres trichent, plus ou moins consciemment (même Gilson, et tant de bonnes âmes)." "

131. Notamment en éreintant dans Itinéraires un pamphlet de Louis Bouyer, « soit dit entre nous, à mon instigation » (lettre au P. Simon, 2 juin 1969).

132. Lettre à J. Oudin, 19 juillet 1969 : «Vous pouvez utiliser cet avis ; De Corte a du poids, et il sait bien sûr que je me sers de ses déclarations. "

133. Lettre à dom Gérard, 27 août 1972. 
possible pour nos amis ${ }^{134}$. $"$ Il pense du bien de Paul Tilloy, auteur d'un opuscule, Le nouvel "Ordo missae ", l'unité dans l'hérésie, qu'il "trouve d'ailleurs très bon ", estimant qu'il s'agit d'un "texte à diffuser » ${ }^{135}$. En revanche, il n'apprécie pas Pierre Lemaire, fondateur de l'Association des pères de famille, puis de la revue Paternité-Maternité. "Pour ce qui est de Pierre Lemaire, je ne vous apprendrai pas que mon jugement à son sujet est, depuis longtemps, sévère : intelligence très médiocre, suffisance fort développée (impossible de discuter avec lui, même avec bonne volonté), absence totale de véritable formation doctrinale ${ }^{136}$. "

Il estime positivement le "sympathique agité [Paul] Scortesco », tout en doutant de ses suppliques au pape : « À quoi ça sert ? C'est malheureusement ainsi que concluent encore trop de traditionnels. Or, de ce côté-là, il me semble que "les jeux sont faits" 137... " En 1972, il penche du même sens : "Lumière est courageux, mais téméraire. Scortesco devient agaçant : dans chaque brochure, il décrit très bien le catastrophique état de l'Église, mais il conclut toujours que, puisque c'est "intolérable", [...] le bon peuple chrétien va se rebeller, et qu'il y aura un grand pape qui, et que (et auquel plus personne n’obéira, la machine étant cassée !) ${ }^{138}$. "

Enfin, la correspondance de Jugnet donne une place importante à une grande figure de l'intransigeantisme romain, celle du cardinal Ottaviani ${ }^{139}$. Jugnet est déçu par les revirements du cardinal. Il invoque favorablement, en juin 1969, un entretien « qui fit quelque bruit ("ce qu'il nous faudrait, c'est un Savonarole"...)» ${ }^{140}$, juste après en avoir décrié un autre : "Interview Ottaviani fort décevante : le bilan du concile est positif pour l'essentiel, etc., etc. Même un peu remis sur pied, il n'est vraiment plus ce qu'il fut et ce qu'il devrait être encore ${ }^{141}$. " Puis, en octobre 1969, il loue sa courageuse défense de la messe :

«Vous avez été comme moi heureux de lire la protestation du cardinal Ottaviani contre le nouvel Ordo missae. Ce ferme langage rappelle l'Ottaviani des anciens jours (et non le "post-conciliaire"...). Puissent de tels textes se multiplier ${ }^{142}$ !"

L'affaire du Bref examen, signé par Ottaviani, duquel il aurait ensuite rétracté sa signature, agace les milieux traditionalistes ${ }^{143}$. Jugnet en est le témoin :

134. Lettre au P. Simon, 7 octobre 1969.

135. Ibid.

136. Ibid.

137. Lettre au P. Simon, 26 avril 1970.

138. Lettre au P. Simon, 6 mars 1972.

139. Sur le premier préfet de la Congrégation pour la doctrine de la foi, voir Em. Cavaterra, Il prefetto del Sant'Offizio : le opere e i giorni del cardinale Ottaviani, Milan, 1990 (Storia e documenti, 100).

140. Lettre au père général des pères de Chabeuil, 9 juin 1969.

141. Lettre au P. Simon, 2 juin 1969.

142. Lettre au P. Simon, 27 octobre 1969.

143. Notamment Jean Madiran, dans "Sur la lettre du cardinal Ottaviani à Paul VI ", dans Itinéraires, supplément au $\mathrm{n}^{\circ} 142$ (avril 1970), p. 1-11. 
«L'affaire Ottaviani-Agustoni-Laffond me dégoûte et me paraît, au fond, sans grande importance, car, outre sa mauvaise vue, le cardinal a déjà tracé des sinusoïdes assez accentuées depuis le concile, il ne faut pas l'oublier. Je pense qu'il n'y a aucun profit à essayer maintenant de faire usage de son nom, de toute manière, tout au plus faut-il secouer les menteurs ${ }^{144} \ldots$ ".

En réponse, le père Simon émet des doutes sur cette rétractation suspecte. Jugnet lui répond : «Je suis bien aise de ce que vous me dites sur l'avis donné au sujet de l'authenticité ou de l'inauthenticité du démenti donné par un pauvre aveugle [Ottaviani] à une de ses déclarations antérieures : au stade où nous en sommes, ça n'a aucune importance réelle ${ }^{145}$. " Ailleurs, il regrette la vigueur passée du cardinal, tout en soulignant la responsabilité de ses familiers : "On me dit que le cardinal Ottaviani a un entourage détestable (du reste, si on voulait faire l'histoire de ses variations ces dernières années, on tracerait une belle sinusoïde...) ${ }^{146}$."

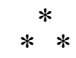

L'étude de la correspondance privée de Louis Jugnet permet ainsi de mettre au jour certaines tensions et certains traits particuliers des réseaux traditionalistes de l'après-concile. La place de Jugnet, pour modeste qu'elle soit, n'est pas négligeable dans des cercles qui l'estiment généralement, bien qu’il s'écarte peu à peu de presque toutes les personnalités laïques, hormis Marcel De Corte. Elle reçoit un nouvel éclairage de ces pièces inédites, qui confirment les textes publiés avant la période étudiée. Le sixième et dernier des cahiers Louis Jugnet est entièrement consacré à « la crise de l'Église ». Les textes qu'il contient ont été diffusés sous forme de notes à l'usage de ses étudiants ou connaissances, entre 1947 et 1968. Pour Jugnet, le nœud du problème n’est ni pastoral ni liturgique, mais doctrinal, et en fin de compte philosophique. Dans ses écrits antérieurs, il y étrille le "nouveau catholicisme », traversant une "crise gravissime ", sous les effets du néo-modernisme. C'est là, nous dit-il, une "maladie de l'intelligence » accompagnée d'une "désacralisation de la religion » : "Le modernisme, même bardé d'exégèse et d'histoire, a une racine philosophique essentiellement. C'est une conception entièrement subjectiviste de la connaissance, un refus du réalisme métaphysique et un nominalisme absolu ${ }^{147}$. " Dès 1947, il évoque une «crise doctrinale effroyable » et un vent nouveau révolutionnaire. Il dénonce des «tentatives constantes pour fonder (?) nos dogmes sur ce que l'évolutionnisme absolu a de plus discuté parmi les savants, un amour intempestif de la philosophie hégélienne, un fanatisme existentialiste, un hyper-bergsonisme, un rationalisme biblique, un laxisme moral, un rejet de la liturgie traditionnelle, etc. [...] Nous revivons (en pire à certains égards, car la défense est moins vigoureuse qu'alors) les années

144. Lettre au P. Simon, 26 avril 1970.

145. Lettre au P. Simon, 18 mai 1970.

146. Lettre au P. Simon, 23 mai 1970.

147. [J. DE Viguerie], «Le mot du président », dans Louis Jugnet, cahier no 6 (1981), p. 8. 
du modernisme condamné par Pie X ${ }^{148}$. »L'encyclique Humani generis de 1950, qualifiée de "très importante », est étudiée à plusieurs reprises, et elle renforce Jugnet dans ses prises de position ${ }^{149}$. Il se plaît à citer, entre autres textes magistériels, une lettre de la Congrégation des études : «Le danger le plus grave aujourd'hui n'est pas celui d'un attachement trop rigide et exclusif à la tradition, mais bien plutôt celui d'un goût exagéré et fort imprudent pour tout ce qui est nouveau, quel qu'il soit ${ }^{150}$. "

En 1951, dans "Église universelle et catholicisme français », il s'attache à recadrer certains de ses compatriotes pour qui «tout l'édifice traditionnel est à refondre entièrement " ${ }^{151}$. Sont brocardés à la fois les conceptions philosophiques non réalistes, les progrès du freudisme et l'évolutionnisme teilhardien. On retrouve les mêmes thèmes dans ses descriptions de la « misère de l'esprit moderniste », ou de la «caractérologie du moderniste et du libéral " ${ }^{152}$. Il part de la philosophie — «une des plus grandes carences »des modernistes — pour descendre aux disciplines les moins élevées : psychologie, psychiatrie, biologie, physique ${ }^{153}$. Douze ans après, il peut s'inquiéter : "Que devient notre christianisme ${ }^{154}$ ? " Il affirme : "C'est à la hiérarchie de prendre les mesures qui sont de son ressort, mais c'est aux fidèles d'argumenter et d'affronter les moyens usuels d'exposition et de discussion, la situation telle qu'ils sont bien forcés de la voir ${ }^{155}$."

Dans les années cinquante, Jugnet se situe dans la mouvance thomiste de l'opposition à la "nouvelle théologie » ${ }^{156}$. En cela, il tient compagnie aux pères dominicains Labourdette, Garrigou-Lagrange, Bruckberger, ou Guérard Des Lauriers ${ }^{157}$. À la fin des années soixante, nous voyons ce philosophe blessé par la crise de l'Église prendre une posture plus entière et plus pugnace face au relativisme doctrinal ou moral, entouré de prêtres désormais margi-

148. L. Jugnet, «Réflexions sur le "nouveau catholicisme" "..., p. 13-14.

149. ID., "L'encyclique et les sciences positives » (1950), édité dans Louis Jugnet, cahier $\mathrm{n}^{\circ} 6$ (1981), p. 37-47, et ID., "L’encyclique Humani generis, Rome et la pensée moderne » (1951), ibid., p. 31-35.

150. ID., "Église universelle et catholicisme français », ibid., p. 19. Nous n'avons pu retrouver le texte cité ici par Jugnet, daté du $1^{\mathrm{er}}$ mai 1951. Néanmoins, ce passage se trouve déjà à l'identique dans une lettre adressée par la Congrégation à l'épiscopat brésilien le 7 mars 1950.

151. Ibid.

152. ID., "De neo modernismo... », p. 49-55, et ID., "Sur la caractérologie du moderniste... », p. $57-62$.

153. Dans "De neo modernismo...", p. 50, Jugnet fustige la figure du "moindre petit clerc inculte et sans armature [qui] se permet de ricaner au nom de l'Ange de l'école ".

154. ID., "Que devient notre christianisme?", édité dans Louis Jugnet, cahier nº 6 (1981), p. 63-69.

155. Ibid., p. 63.

156. Jugnet avait reçu de Pie XII une « chaleureuse et longue lettre de félicitations » pour son ouvrage Pour connaître la pensée de saint Thomas d'Aquin, Paris, 1949 (Pour connaître...) [rééd. [Paris], 1964 ; Paris, 1975 ; Bordeaux, 1979 ; Paris, 1999].

157. Parmi de nombreuses publications, on peut mentionner l'article programmatique du R. P. R. Garrigou-Lagrange, "La nouvelle théologie, où va-t-elle? ", dans Angelicum, $\mathrm{n}^{\circ} 23$ (juil.-déc. 1946), p. 126-145, le débat entre jésuites et dominicains présenté par les PP. M. Labourdette, M.-J. Nicolas, R.-L. Bruckberger, Dialogue théologique : pièces du débat entre "La revue thomiste", d'une part, et les RR. PP. de Lubac, Daniélou, Bouillard, Fessard, von Balthasar, s.j., d'autre part, Saint-Maximin, 1947, ou encore l'opuscule du P. M. Labourdette, Foi catholique et problèmes modernes : avertissements et directives du souverain pontife, [Tournai, 1953] (Le monde et la foi). 
nalisés ou proscrits. Les principes demeurent, les formes de son combat évoluent. Hormis l'opposition à Vatican II et à la "nouvelle messe », et pour cause, ses lettres témoignent d'une véritable continuité spéculative, élaborée au sortir de la guerre. La doctrine intransigeante qu'il prêche reste identique (il allègue saint Vincent de Lérins : "Crescat igitur, sed eodem sensu»), fondée sur un thomisme « strict », mais la manière de la prêcher se renforce, se durcit, s'étend et se retourne même contre une papauté accusée d'abandonner le combat. Dorénavant, ce n'est plus soutenu par le pape, mais bien souvent malgré le pape, voire contre le pape, qu'il agit, entendant conserver une ligne de conduite inflexible ${ }^{158}$.

Cette réaction lui assurera sa réputation, ainsi que des félicitations venues de l'étranger. D'Italie, Carlo Meli, de la revue Relazioni, l'informe du projet de création d'un Institut d'études politiques, chargé « de combattre la puissance écrasante de la gauche progressiste et de créer des cadres authentiquement catholiques, dans le respect des valeurs traditionnelles ", et le sollicite en tant que « figure représentative de l'orthodoxie catholique ». Du Brésil, sous la plume de Fabio Vidigal Xavier da Silveira, membre du conseil national de l'association Tradition, famille, propriété, il reçoit un témoignage de «sympathie pour la lutte [qu'il] condui[t] en défense de la vraie doctrine de notre sainte Eglise catholique " ${ }^{159}$. D'autres témoins viendront apporter leur soutien posthume, comme Gustavo Daniel Corbi, qui publiera depuis l'Argentine Tres maestros : Billot, Jugnet, Meinvielle (Buenos Aires, 1980), et évoquera " un maître authentique » 160 .

En France, de nombreux disciples ou amis venus de multiples horizons feront montre d'une gratitude intellectuelle. Dès sa mort, la revue L'ordre français publie un numéro spécial qui lui est consacré, avec des contributions du père Georges Delbos, m.s.c., de Marcel De Corte (université de Liège), de René Giraudon (lycée Pierre-de-Fermat), du $\mathrm{D}^{\mathrm{r}}$ François Lamasson, de Jean de Quissac (institut d'études politiques de Toulouse) et de Jean de Viguerie (université d'Angers). Ce dernier présida ensuite une Association des Amis de Louis Jugnet, qui publia six cahiers Louis Jugnet (1975-1981), regroupant à la fois des textes inédits du maître et des témoignages de ses disciples, dont celui de Pierre Mesnard (université de Poitiers). Plus tard, Jean Bastier (université de Toulouse) ${ }^{161}$, Pierre Manent (École des hautes études en sciences sociales) ${ }^{162}$, ou encore l'abbé Claude

158. Il n’y a cependant aucune velléité schismatique chez Jugnet, seulement des attaques ponctuelles et tactiques contre le pape, qu'il sait aussi défendre, comme le montre son élogieuse "Note sur l'encyclique Ecclesiam suam de Paul VI, 6 août 1964 (Transfiguration) », éditée dans Louis Jugnet, cahier $n^{\circ} 6$ (1981), p. 77-81, jugeant, p. 81, "assez manifeste que le pape a voulu "court-circuiter" la poussée moderniste et progressiste à la prochaine session du Concile ».

159. Lettre de F. Vidigal Xavier da Silveira, 13 août 1970.

160. G. D. Corbi, "Un maître authentique : Louis Jugnet vu d'Argentine ", dans Le sel de la terre, $\mathrm{n}^{\circ} 47$ (hiver 2003-2004), p. 140-171.

161. J. BAstien, "Conversations avec Louis Jugnet (printemps 1962) », dans Cahiers de Chiré, $\mathrm{n}^{\circ} 11$ (1996), p. 233-24l [repris dans Lecture et tradition, nouv. série, no 25 (mai 2013), p. 12-19].

162. P. Manent, Le regard politique : entretiens avec Bénédicte Delorme-Montini, [Paris], 2010, qui affirme notamment, p. 33-34: «Voilà un homme, Jugnet, qui rassemblait en sa personne tous les traits qui aujourd'hui suscitent le rejet immédiat : catholique traditionnel, maurrassien, 
Barthe ${ }^{163}$ exprimeront leur dette à l'égard de Jugnet. Dans la mouvance de la Fraternité sacerdotale Saint-Pie-X, l'héritage de Louis Jugnet est aussi vivace. La revue Le sel de la terre, éditée par les dominicains d'Avrillé, lui a consacré en 2003-2004 un numéro spécial avec une bibliographie commentée ${ }^{164}$, et en 2009 s'est tenu à l'Institut universitaire Saint-Pie-X un colloque intitulé "Actualité de Louis Jugnet ", dont les actes ont paru dans la revue $V u$ de haut ${ }^{165}$. Enfin, depuis 2013, les éditions Diffusion de la pensée française ont réédité ses principales œuvres et publié un « Hommage à Louis Jugnet » dans leur «bulletin littéraire contrerévolutionnaire », Lecture et tradition ${ }^{166}$.

Lui qui était à la fin de sa vie « étonné, et édifié, de l'extrême réceptivité de beaucoup " de ses derniers élèves, trouvant "encore plus d'ouverture et de rectitude chez eux que chez ceux d'il y a dix ans " ${ }^{167}$, ne s'était pas trompé à leur sujet. À travers la mémoire transmise par ses disciples directs, les réseaux intellectuels catholiques intransigeants, dans leur mouture actuelle, continuent de s'abreuver de la pensée de ce philosophe engagé.

\author{
Cyrille Dounot, \\ université d'Auvergne, \\ Centre Michel-de-L'Hospital.
}

et qui était en même temps un enseignant incomparable et un des hommes les plus dignes d'estime que j'aie connus."

163. Voir P. Airiau, "Des théologiens contre Vatican II (1965-2005)", dans D. Avon et M. Fourcade (éd.), Un nouvel âge de la théologie? 1965-1980. Colloque de Montpellier, juin 2007, Paris, 2009 (Signes des temps), p. 74-75.

164. "Anniversaire : Louis Jugnet (1913-1973)", dans Le sel de la terre, no 47 (hiver 20032004), p. 130-236.

165. "Actualité de Louis Jugnet : actes du colloque du 17 octobre 2009 ", dans Vu de haut, $n^{\circ} 16$ (printemps 2010), p. 3-137. Outre les hommages de Michel Brignol et de Jean de Viguerie, on peut citer les contributions de l'abbé Alain Lorans, "Ce que Louis Jugnet nous apporte aujourd'hui » (p. 35-48), et de l'abbé Philippe Bourrat, "Louis Jugnet, un guide pour notre temps : la question du modernisme » (p. 49-68).

166. "Doctrines philosophiques et systèmes politiques : hommage à Louis Jugnet (19131973) ", dans Lecture et tradition, nouv. série, no 25 (mai 2013), p. 1-26. Les trois ouvrages de Louis Jugnet réédités par les éditions Diffusion de la pensée française sont Doctrines philosophiques et systèmes politiques, Chiré-en-Montreuil, 2013 ; Problèmes et grands courants de la philosophie, Chiré-en-Montreuil, 2013, et Catholicisme, foi et problème religieux, Chiré-enMontreuil, 2014.

167. Lettre au P. Simon, 19 novembre 1970. 
